\title{
Radial velocities of open stellar clusters: A new solid constraint favouring Tsallis maximum entropy theory
}

\author{
J.C. Carvalho ${ }^{\text {a }}$, B.B. Soares ${ }^{\mathrm{a}}$, B.L. Canto Martins ${ }^{\mathrm{a}}$, J.D. do Nascimento Jr. ${ }^{\mathrm{a}}$, \\ A. Recio-Blanco ${ }^{\mathrm{b}}$, J.R. De Medeiros ${ }^{\mathrm{a}, *}$ \\ ${ }^{a}$ Departamento de Física, Universidade Federal do Rio Grande do Norte, 59072-970 Natal, RN, Brazil \\ ${ }^{\mathrm{b}}$ Departmento Cassiopée, UMR 6202, Observatoire de la Côte d'Azur, B.P. 4229, F-06304, Nice, Cedex 4, France
}

Received 19 April 2007

Available online 24 May 2007

\begin{abstract}
In the present study we apply a Tsallis maximum entropy distribution law to the study of the stellar residual radial velocity in a sample of 13 stellar open clusters. From a comparison between results obtained from the analysis based on Tsallis law and on the one based on the Maxwellian law we show that the generalized Tsallis distribution fits more closely the observed distribution of the stellar residual radial velocities for these stellar clusters. We have found clear evidences that the $q$-parameter in the Tsallis generalized distribution depends on stellar cluster ages for clusters older than $10^{9} \mathrm{yr}$. There is also some indication that $q$ increases with cluster galactocentric distance. The results obtained in this work represent an additional solid constraint in the stellar astrophysics favoring the Tsallis maximum entropy theory.
\end{abstract}

(C) 2007 Elsevier B.V. All rights reserved.

Keywords: Stars: rotation; Distribution; Main sequence; Open cluster

\section{Introduction}

The nature of the statistical laws controlling the distribution of different stellar physical parameters, in particular rotational and radial velocities, is not well established yet, in spite of a conventional practice which seeks explanations based on Gaussian and Maxwell-Boltzmann distribution or on analytical functions with no clear physical motivation. For instance, a preliminary study by Soares et al. [1] shows that these functions cannot conveniently fit the observed distribution of projected rotational velocity of stars in the Pleiades stellar open cluster. In fact, the nature of such a distribution is better explained on the basis of a Tsallis maximum entropy distribution function.

In the present study we show that the question of the nature of the distribution of stellar radial velocity in stellar open clusters, as in the case of projected rotational velocity, is not simply a problem of which mathematical function model to use, but it depends primarily on which statistical mechanics is used.

*Corresponding author.

E-mail address: renan@dfte.ufrn.br (J.R. De Medeiros). 
Open clusters are physically related group of stars held together by their mutual gravitational attraction. They are very important laboratories in the study of stellar physics because, in particular, their member stars have similar age and chemical composition. Thus, the behaviour of other subtle physical variables or properties are much easily studied than they are for isolated field stars. The knowledge of different properties of open clusters, as the distribution of dispersion velocity and phase density, is necessary to determine the type of relaxation mechanism operating in the system during its evolution. There are three main mechanisms: collisional relaxation, where a Maxwellian distribution is attained; the Lynden-Bell violent relaxation, leading to a Fermi distribution, which corresponds to a constant phase density for energies lower than a certain maximum value; and, the third one, a relaxation associated with radial orbit instability, that leads to a nonmonotonic function (e.g. Refs. [2-4]). Observations of star samples in the solar neighbourhood point to different laws for velocity dispersion increasing with the stellar age, including discontinuities in the local velocity distributions, but the nature of such a phenomenon is not well understood yet (e.g. Ref. [5]).

Following the same procedure used by Soares et al. [1] we study the behaviour of the distribution of radial velocity of stars located in 13 stellar open clusters. For such a purpose we have used the Tsallis Maximum Entropy distribution applied in the study of the observed rotational velocity distribution of the stars in the Pleiades cluster. This new distribution function (hereafter $q$-maxwellian) generalizes the Maxwell-Boltzmann function [6], and arises from the non-extensive statistical framework first proposed by Tsallis [7] and applied successfully to different astrophysical problems, like matter distribution of self-gravitating system [8-12], stellar polytropes [13], Lévy and correlated-like anomalous diffusions [14], solar neutrino fluxes [15], cosmological model [8], dynamic linear response theory [16], velocity distribution of galaxy clusters [9], gravothermal catastrophe [17] and Jeans gravitational instability [18]. As shown by Tsallis et al. [19], in the generalized thermostatistics, the ubiquity and robustness of Lévy distributions in nature follow naturally from the generalized central limit theorem. In this context, Normal, Gaussian-type, diffusion and the so-called anomalous, Lévy-type, superdiffusion can now be unified in a single picture. The reader is also referred to Tsallis [20] and Abe and Okamoto [21] for a more enlarged discussion on Tsallis nonextensive statistics and its applications.

In Section 2 we describe the sample and how we selected stars from the original database. In Section 3 we determine the best fit for the observed distribution of residual radial velocity $V_{r}$ for four stellar clusters. Section 4 brings a discussion of the results. Finally, the conclusions and perspective of future studies are presented in Section 5.

\section{The stellar sample}

For this study we have selected 13 stellar clusters that in the literature represent the open clusters with the largest number of component stars with measured radial velocity. This is an important requirement

Table 1

Physical characteristics of stellar clusters used in this work

\begin{tabular}{lccc}
\hline Cluster & Distance $(\mathrm{pc})$ & $\log$ Age $(\mathrm{yr})$ & Metallicity \\
\hline Pleiades & 150 & 8.131 & -0.11 \\
NGC 1039 & 499 & 8.243 & -0.30 \\
NGC 6475 & 301 & 8.475 & +0.14 \\
NGC 2099 & 1383 & 8.540 & +0.08 \\
Mel 111 & 96 & 8.652 & -0.06 \\
Praesepe & 187 & 8.863 & +0.14 \\
Hyades & 45 & 8.896 & +0.17 \\
NGC 0752 & 457 & 9.050 & -0.08 \\
NGC 7789 & 2337 & 9.235 & -0.08 \\
NGC 6939 & 1185 & 9.346 & +0.02 \\
M67 & 908 & 9.409 & -0.05 \\
NGC 6791 & 5853 & 9.643 & +0.15 \\
Ber 39 & 4780 & 9.900 & -0.17 \\
\hline
\end{tabular}


considering that for our purpose we need to work with statistically robust data samples. In addition, the selected clusters represent an age sequence that constitute an important constraint for an evolutionary study of the distribution of the radial velocity, one of the main goals of the present study. The main physical characteristics of the selected stellar clusters are displayed in Table 1.

For all the clusters, distances, ages and radial velocities were taken from the WEBDA data basis (e.g. Ref. [22]). Metallicities were taken from Refs. [22-26].

An important step in the present analysis was the definition of the stars members of each cluster suitable for our purposes. First, we have removed all the stars presenting binarity signatures so that the radial velocity would not be affected by their orbital motion.

Second, because the sample of stars listed in the literature as cluster members present some misidentifications, we have tried to clean more properly the sample by applying the following two methods to discriminate between member and non-member stars.

The first uses the membership probability given by the SIMBAD database (operated at Centre de Donnes Stellaires, Strasbourg, France) to eliminate those stars whose probability is less than 0.9. There are, however, many stars with no probability available and in these cases we arbitrarily attribute probability 1.0 to them. This method does not seem to correctly discriminate well between members and no members of the clusters since there is still a large number of stars with velocity far from the central peak of the distribution while many others near the peak have been selected out.

In the second method we reject stars whose difference between the star velocity and the average velocity is greater than three times the standard deviation of the distribution. We apply this procedure iteratively until it converges, which happens when the number of stars left becomes constant. The process converges after typically six iterations and is shown in Fig. 1 for four typical clusters. Here and forth we select four clusters,

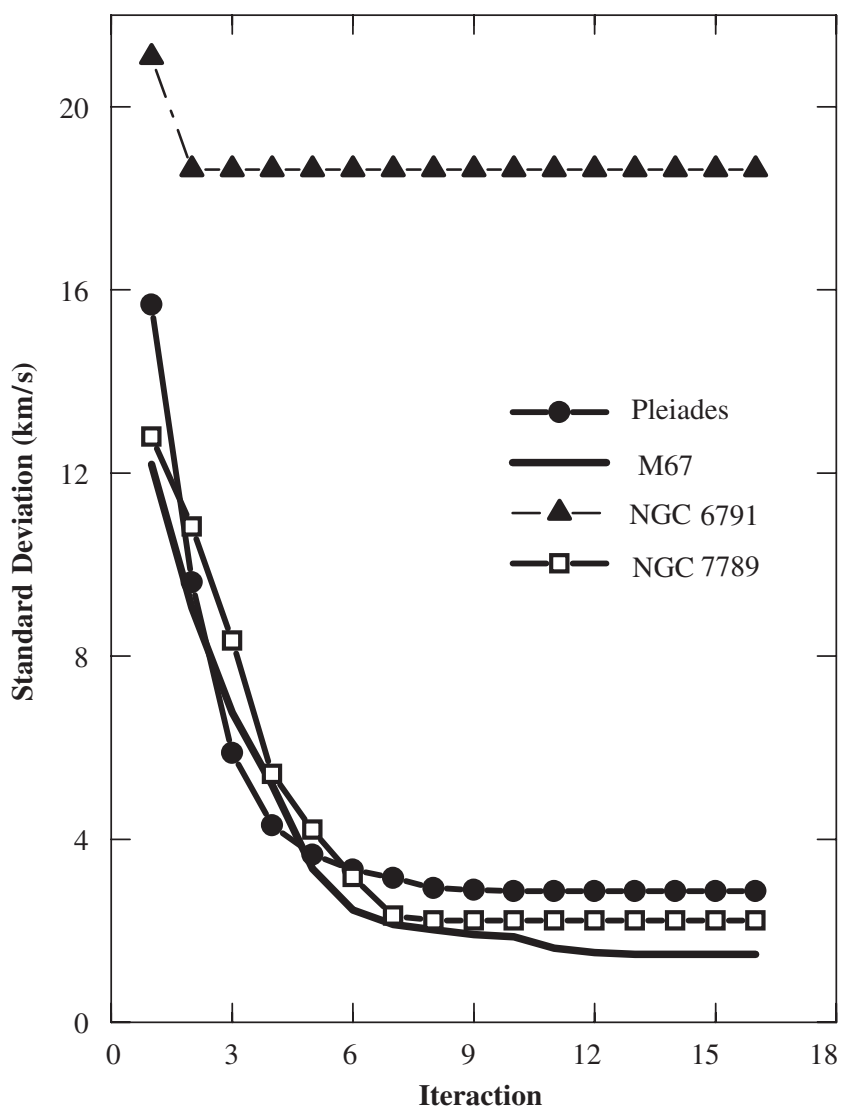

Fig. 1. Value of the standard deviation of the velocity distribution as a function of the number of iteration for the second selection method as described in the text. 
Table 2

Best $q$ and $\sigma_{q}$-values determined using Kolmogorov-Smirnov test for the clusters in our sample

\begin{tabular}{|c|c|c|c|c|c|}
\hline Cluster & $N_{t}$ & $N_{3 \sigma}$ & $q$ & $\sigma_{q}$ & $P_{\max }$ \\
\hline Pleiades & 383 & 323 & $2.24_{-0.22}^{+0.20}$ & 1.31 & 0.89 \\
\hline NGC 1039 & 44 & 42 & $1.82_{-0.69}^{+1.76}$ & 3.13 & 0.87 \\
\hline NGC 6475 & 94 & 89 & $1.75_{-0.33}^{+0.33}$ & 4.59 & 0.55 \\
\hline NGC 2099 & 56 & 55 & $2.49_{-0.31}^{+0.36}$ & 2.10 & 0.52 \\
\hline Mel 111 & 119 & 111 & $1.46_{-0.14}^{+0.18}$ & 12.22 & 0.28 \\
\hline Praesepe & 286 & 231 & $2.29_{-0.17}^{+0.15}$ & 1.23 & 0.78 \\
\hline Hyades & 418 & 352 & $1.78_{-0.12}^{+0.07}$ & 3.20 & 0.32 \\
\hline NGC 0752 & 42 & 42 & $1.38_{-0.38}^{+0.32}$ & 16.69 & 0.27 \\
\hline NGC 7789 & 123 & 99 & $1.65_{-0.44}^{+0.34}$ & 1.59 & 0.87 \\
\hline NGC 6939 & 41 & 39 & $2.22_{-0.48}^{+0.66}$ & 1.35 & 0.95 \\
\hline M67 & 220 & 165 & $2.05_{-0.36}^{+0.31}$ & 0.86 & 0.86 \\
\hline NGC 6791 & 55 & 54 & $2.69_{-0.60}^{+1.01}$ & 5.04 & 1.00 \\
\hline Ber 39 & 44 & 39 & $3.82_{-1.24}^{+4.17}$ & 2.49 & 0.97 \\
\hline
\end{tabular}

namely, Pleiades, M67, NGC 6791 and NGC 7789, to illustrate the results. The relevant information and results on all 13 clusters is given in Table 2.

The resulting distributions, shown in Fig. 2, strongly suggests that the distributions are far from being Gaussian. For instance, Soares et al. [1] have already shown that the rotational velocity distribution of the Pleiades is not a classical Maxwellian but can be fitted by the Tsallis maximum entropy distribution law. In the following we carry out a Kolmogorov-Smirnov test in order to quantitatively investigate whether this also applies to the radial velocity distribution.

\section{Numerical results}

The generalized $q$-Gaussian distribution function for the radial velocity $v_{r}$ can be written as

$$
\Phi_{q}\left(v_{r}\right)=A_{q}\left[1-(1-q) \frac{v_{r}^{2}}{\sigma_{q}^{2}}\right]^{1 /(1-q)},
$$

where $\sigma_{q}$ is the characteristic width, $q$ is a free parameter and $A_{q}$ is a constant. In the limit when $q=1$ the above distribution should reproduce the standard Gaussian distribution

$$
\phi\left(v_{r}\right)=A \exp \left(-\frac{v_{r}^{2}}{\sigma^{2}}\right) .
$$

We then tried to fit both distributions to the observational data. In addition, in order to avoid biases in constructing the frequency histograms due to arbitrary choices of bin width, we have instead studied the observed cumulative distribution of the residual radial velocities. We then used the Kolmogorov-Smirnov statistical test to obtain the best $\Phi_{q}\left(v_{r}\right)$ and consequently the best $q$-value and $\sigma_{q}$-value for each observed cumulative distribution. The results are shown in Table 2 where we give the best fit value for the parameters $q$ and $\sigma_{q}$, the maximum probability $P_{\max }$ for the pair $\left[q-\sigma_{q}\right]$, the total number $N_{t}$ of stars in each sample, the number of stars in the analysed sample $N_{3 \sigma}$ after selection using the iterative $3 \sigma$ method as described in Section 2 and the age of the cluster.

In Fig. 3 we show the logarithm of the observed normalized distributions of the residual radial velocity (dots), the Gaussian best fit (dashed line) and the generalized $q$-Gaussian distribution (full line) for the best fit parameters given in Table 2 as a function of $v_{r}^{2}$. In all the cases the difference between the standard Gaussian distribution and the generalized $q$-Gaussian is quite evident with the latter being able to fit the high velocity queue of the observed radial velocity distribution. In Fig. 4 we present the behaviour of the parameter $\sigma_{q}$, the characteristic width of the $q$-Gaussian, as a function of the parameter $q$. It is clear that, at least for the Pleiades 

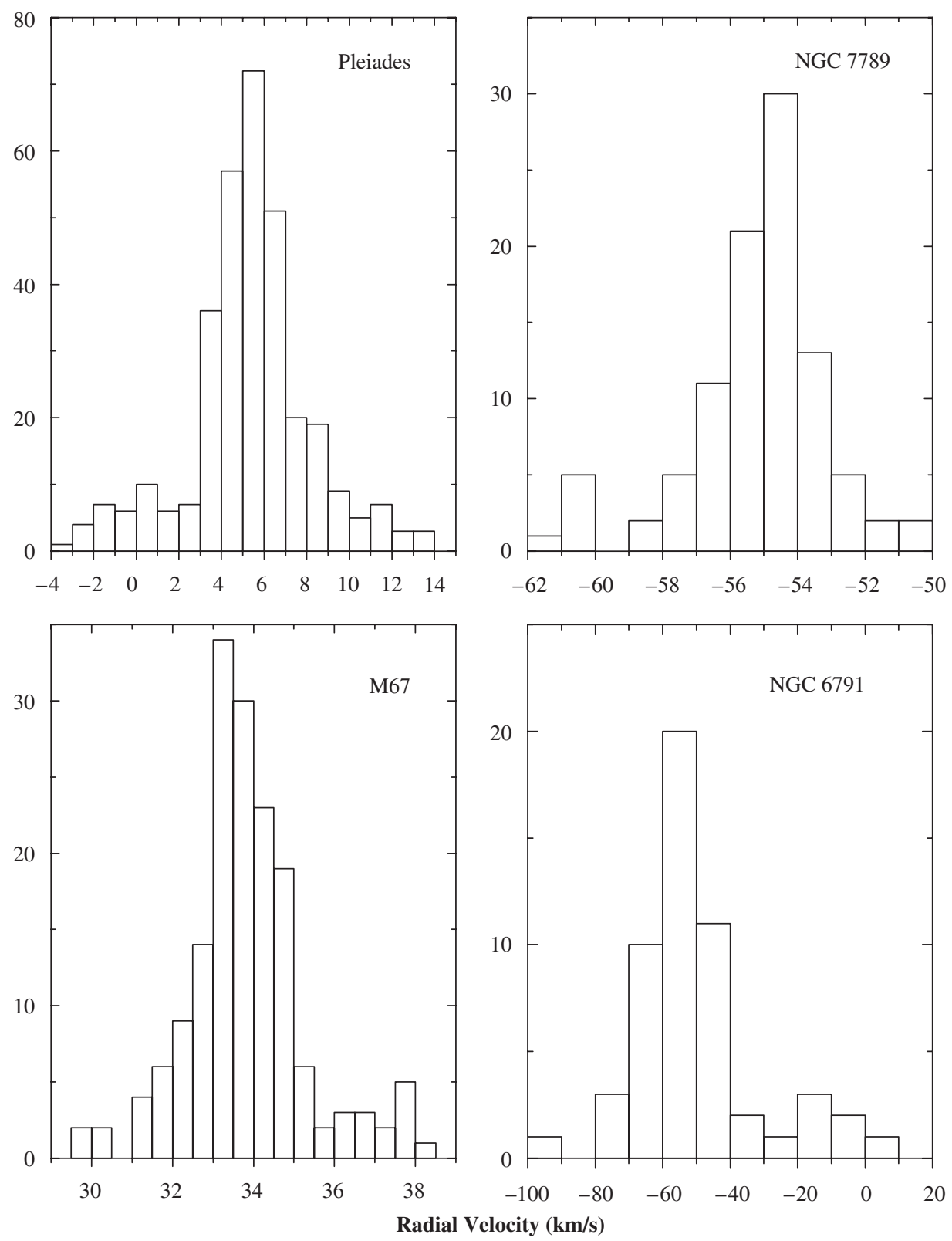

Fig. 2. Radial velocity histogram of four stellar clusters after removing stars according to the second selection method, that is, star velocity difference to the central peak of the distribution larger than $3 \sigma$.

sample, the standard Gaussian $(q=1)$ is in the rejection region or outside the curve which delineates 0.05 significance level. For the other clusters, the Gaussian lies near the frontier of the 0.25 significance level curve but still visibly detached from the $q$-Gaussian. Finally in Fig. 5 we have plotted the fitted value of the $q$-parameter against the age of the cluster. The error bars correspond to a 0.05 confidence limit as given in Table 2.

\section{Discussion}

We have performed an analysis of the main dependence of $q$ on other main parameters of the sample clusters such as galactic coordinates, galactocentric distance, age and total visual magnitude. We have run a 

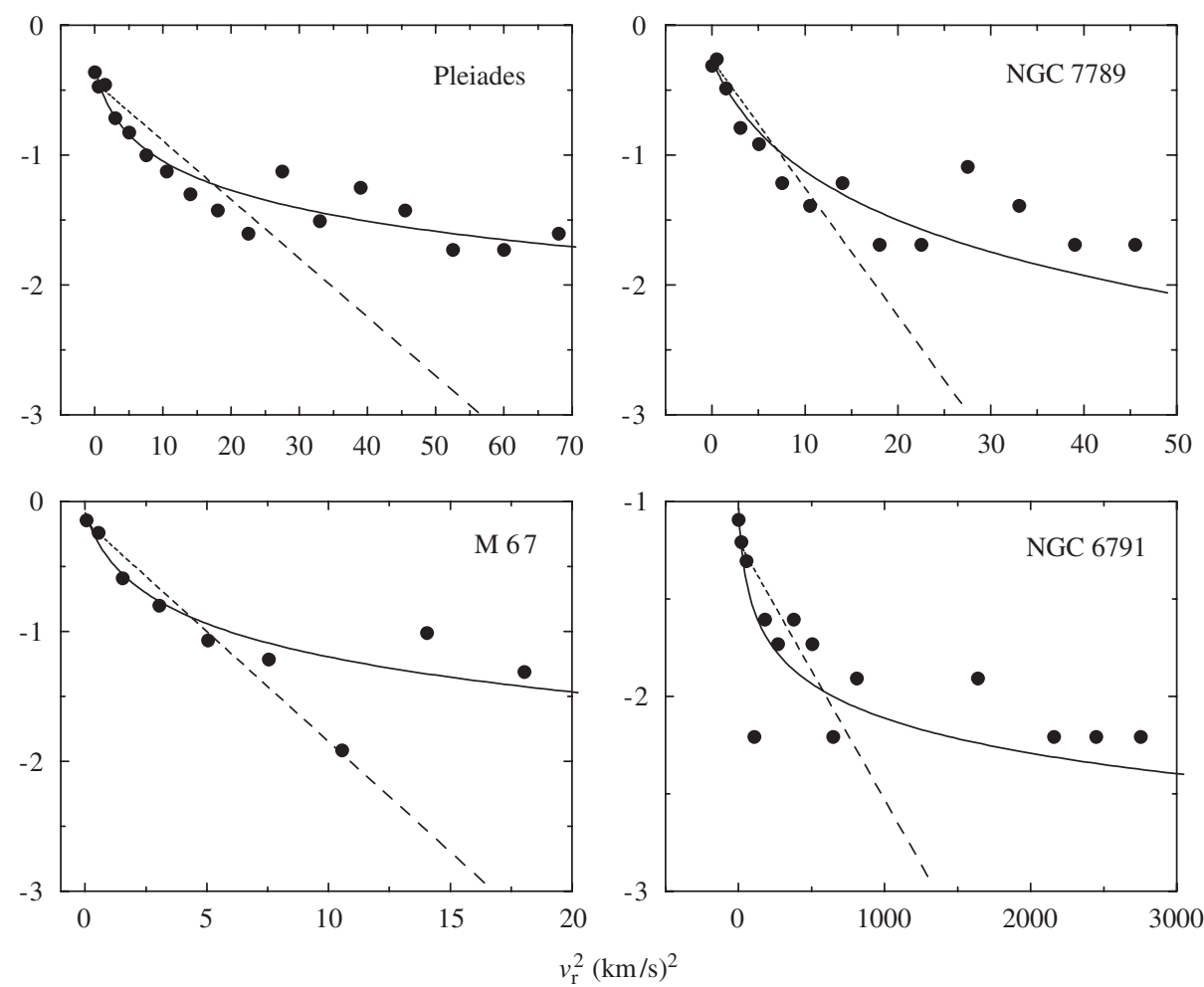

Fig. 3. Comparison between the logarithm of the observed (dots) and fitted Gaussian (dashed line) and $q$-Gaussian (full line) distribution of residual radial velocity of four stellar open clusters.
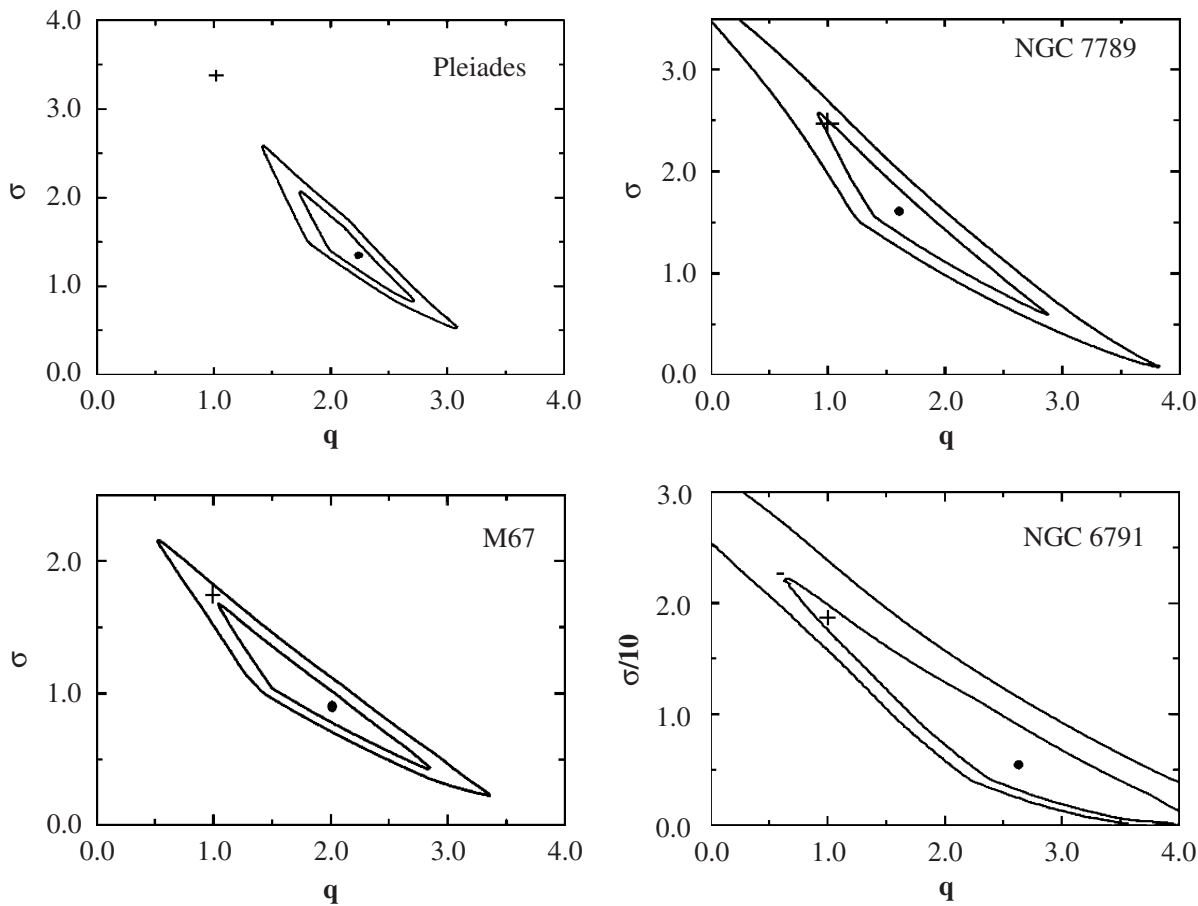

Fig. 4. The rejection region (outside the curve) of the null hypothesis that the $v_{r}$ distribution is drawn from the $q$-Gaussian function at 0.05 significance level (outmost curve) and at 0.25 significance level (innermost curve) for stars in four clusters. The cross ( + ) represents the best $\sigma$ for the standard Gaussian function $(q=1)$ and the $\operatorname{dot}(\bullet)$ represents the maximum probabilities for $q$ and $\sigma_{q}$ values. 


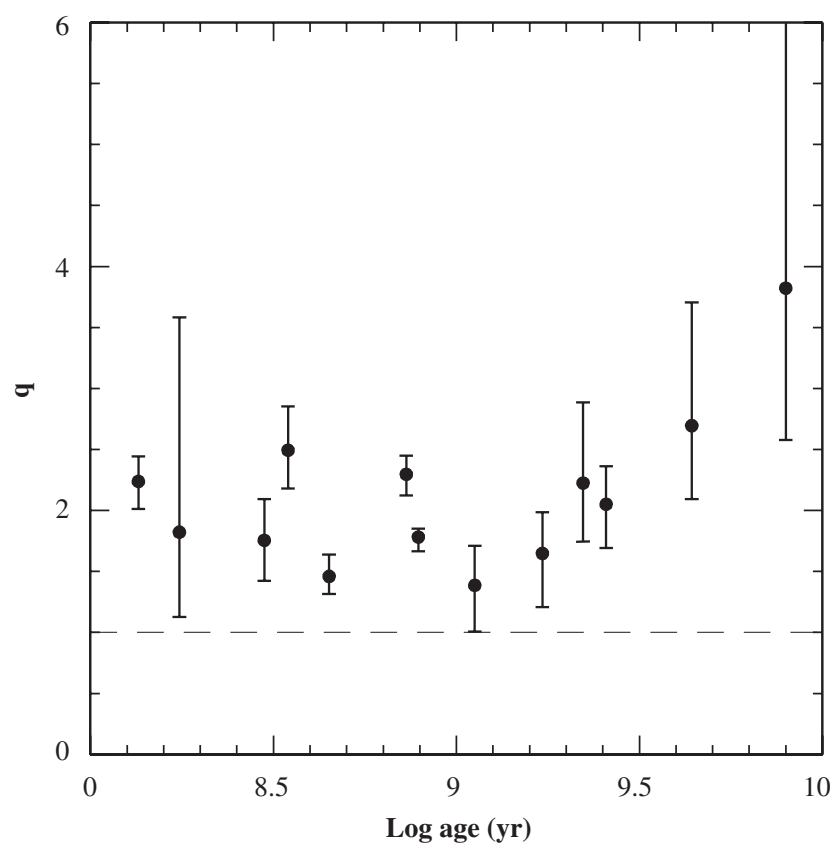

Fig. 5. Value of the fitted parameter $q$ as a function of the cluster age.

Principal Component Analysis programme and found that the sample has dimensionality 2 or 3 (as there are 3 eigenvalues out of 6 which are different from zero). Two of them are higher than 1, indicating that they carry out most of the information. That means that 2 or 3 parameters from those considered should be enough to explain the major part of the sample variance.

The correlation between $q$ and age is $r=0.46$ which means that age seems to explain only about $21 \%$ of the variation of $q$, with older clusters having a higher $q$ value. This is also seen in Fig. 5 where the dependence of $q$ on age, although small in the range $10^{8}-10^{9} \mathrm{yr}$, it is quite clear for clusters older than $10^{9} \mathrm{yr}$. Of course, this is only a crude and preliminary analysis since we do not take into account the errors and the small statistics.

On the other hand, it is interesting to note the important dependence of the $q$ parameter on the distance to the galactic centre. The correlation is between 0.70 and 0.77 depending on the clusters included in the sample, that is, with or without Mel 111 and/or NGC 6791. That means that the distance to the galactic centre seems to account for $\sim 50 \%$ of the $q$ parameter variance, with clusters further from the galactic centre having a higher value of $q$.

The variation that we find with the total cluster luminosity is not very significant, but it is in fact the third parameter by order of importance in what correlation is concern. The correlation between the total visual magnitude and $q$ is $r=0.23$ (about $6 \%$ of the total variance of $q$ ). The total visual magnitude can in fact be an indirect indicator of the total mass of the cluster.

The observed correlation between the $q$-parameter and cluster age, galactocentric distance and total cluster luminosity, all add up together to make us confident that the significant deviation of the residual radial velocity distribution of our open cluster sample from a standard Maxwellian is a result of complex dynamical relaxation processes. Not only age but also the environment seems to play an important role. For instance, the great majority of young clusters $\left(<10^{9} \mathrm{yr}\right)$ is located at the vicinity of the Sun near the galactic plane while old ones are far from the Sun and the galactic disc. Older clusters could have cross the disc more times so that one expects important gravitational disturbances that would affect their velocity distribution [27].

In the case of globular clusters, this has already been discussed in the literature. For example, according to both theory [28] and simulations for globular clusters [29], the rate of evaporation of the stars from a stellar cluster is determined almost completely by its mass and its distance from the galactic centre. Also, globular clusters evolved dynamically (1) by ejection of stars as a result of relaxation through stellar encounters 
("evaporation") and (2) loosening of the cluster by tidal shocks due to its passages through the galactic plane and through the high-force region of perigalacticon.

These considerations could well be applied to our open cluster sample since, as shown in this work, the value of the $q$-parameter of the Tsallis distribution of radial velocity seems to have a clear dependence on age, distance from the galactic centre and luminosity/mass.

\section{Conclusions}

The present study brings the results of a statistical analysis of the residual radial velocity of a sample of 13 open stellar clusters, with ages in a range spanning two decades $\left(10^{8}-10^{10} \mathrm{yr}\right)$. The distribution of the observed residual radial velocity for these stellar clusters are far from being described by a classical Gaussian. The $q$-Maxwellian distribution function described in the context of the Tsallis maximum entropy theory is clearly the more feasible function to fit the distribution of this important stellar observable, independent of the cluster age.

Another important aspect of the present analysis is the clear trend for a dependence of the $q$-parameter on the cluster age above $10^{9} \mathrm{yr}$ and distance from the galactic centre. We find an indication that the older and more distant from the centre the stellar cluster is, the more the distribution of residual radial velocity deviates from classical Gaussian or Maxwellian functions. This finding seems to point for the fact that, in astrophysical systems where dispersion of a parameter increases with age, as is the case for stellar cluster's radial velocity (e.g. Refs. [2,30]) and there is a strong influence of the environment, Tsallis maximum entropy theory is the most suitable to explain the distribution of such a parameter because here, differently from classical Gaussian and Maxwellian functions, the $q$-parameter follows also the time evolutionary behaviour of the distribution.

Regarding the physical meaning of the $q$-parameter, we have witnessed a quite constructive debate on this theme. In our case there are indications that it is related to the fact that the systems we have analysed are in a non-equilibrium state, namely far from a gravitational relaxed state. For instance, preliminary investigation we are now developing on the distribution of radial velocity of stellar globular clusters, which surely are in a gravitationally relaxed state, shows that the $q$-parameter is a measure of the level of departure from equilibrium. That is most likely the reason why our results, in the case of open clusters, show a large range of values of $q$, in most cases, different from 1 . This and other questions we intend to address in a forthcoming paper.

\section{Acknowledgements}

This work has been supported by continuous grants from the CNPq Brazilian Agency. It is a pleasure to thank the two anonymous referees for their remarks and suggestions for improvements of the present paper.

\section{References}

[1] B.B. Soares, J.C. Carvalho, J.D. do Nascimento Jr., J.R. De Medeiros, Physica A 364 (2006) 413.

[2] J. Binney, S. Tremaine, Galactic Dynamics, Princeton University Press, Princeton, NJ, 1994.

[3] T.K. Nakamura, Astrophys. J. 531 (2000) 739.

[4] T.E.C. Merrall, R.N. Henriksen, Astrophys. J. 595 (2003) 43.

[5] S. Alcobz, R. Cubarsi, Astron. Astrophys. 442 (2005) 929.

[6] R. Silva, A.R. Plastino, J.A.S. Lima, Phys. Lett. A 249 (1998) 401.

[7] C. Tsallis, J. Stat. Phys. 52 (1988) 479.

[8] V.H. Hamity, D.E. Barraco, Phys. Rev. Lett. 76 (1996) 4664.

[9] A. Lavagno, G. Kaniadakis, M. Rego-Monteiro, P. Quarati, C. Tsallis, Astr. Lett. Commun. 34 (1998) 449.

[10] A.R. Plastino, A. Plastino, Phys. Lett. A 174 (1993) 384.

[11] A.R. Plastino, Europhys. News 36 (2005) 208.

[12] A. Taruya, M. Sakagami, MNRAS 364 (2005) 990.

[13] B. Boghosian, Phys. Rev. E 53 (1996) 4754.

[14] D.H. Zanette, P.A. Alemany, Phys. Rev. Lett. 75 (1995) 366.

[15] G. Kaniadakis, A. Lavagno, P. Quarati, Phys. Lett. B 369 (1996) 308.

[16] A.K. Rajagopal, Phys. Rev. Lett. 76 (1996) 3469. 
[17] A. Taruya, M. Sakagami, Physica A 307 (2002) 185.

[18] J.A.S. Lima, R. Silva, J. Santos, Astron. Astrophys. 396 (2002) 309.

[19] C. Tsallis, S.V.F. Lévy, A.M.C. Souza, R. Maynard, Phys. Rev. Lett. 75 (1995) 3589.

[20] C. Tsallis, Braz. J. Phys. 29 (1999) 1.

[21] S. Abe, A. Okamoto (Eds.), in: Nonextensive Statistical Mechanics and Its Applications, Springer-Verlag, Heidelberg, 2001.

[22] D. Egret, M.A. Albrecht (Eds.), Information and On-Line Data in Astronomy, Kluwer, Dordrecht, pp. $137-138$.

[23] M. Grenon, Astrophys. Space Sci. 265 (1/4) (1991) 331.

[24] S. Bartasiute, G. Tautvaisiene, Astrophys. Space Sci. 294 (2004) 225.

[25] J.L. Marshall, Ch.J. Burke, D.L. DePoy, A. Gould, J.A. Kollmeier, Astron. J. 130 (2005) 1916.

[26] B.J. Taylor, Astron. Astrophys. 377 (2001) 473.

[27] H.J.G.L.M. Lamers, M. Gieles, A\&A 455 (2006) 17.

[28] I.R. King, Astrophys. J. 71 (1996) 64.

[29] E. Vesperini, D.C. Heggie, MNRAS 289 (1997) 898.

[30] D. Mihalas, J. Binney, W.H. Freeman and Co, San Francisco, CA, 1981. 